\title{
The Complex of Lines from Successive Points and the Horopter
}

\author{
J.M. Selig
}

\begin{abstract}
The Horopter is the set of points in space which project to the same image points in the two cameras of a stereo vision system. Modern proofs are given for many of the classical results about the Horopter. Some of these rely on another classical construction, the quadratic complex of lines joining successive points. A modern derivation of this is also given. In particular the circumstances in which the image of the Horopter can degenerate is discussed in some detail. Finally, these ideas are extended to the case of optical flow where there is a single camera observing the velocities of points in space.
\end{abstract}

\section{INTRODUCTION}

In binocular or stereo vision, using a pair of identical cameras, the points in space which produce image points in both cameras with the same image coordinates lie on a curve known as the Horopter. This term seems to have been introduced by Franciscus Aguilonius in the second of his six books in optics in 1613, however the term was used differently there.

In modern times the horopter was first studied by Helmholtz in his "Handbuch der Physiologischen Optik" in the mid 1800s [4]. It is Helmholts who is responsible for the main results of the geometry of the Horopter, for example, in general the Horopter is a twisted cubic curve.

These ideas seem to have been first discussed in the computer vision literature by Maybank in [9] in connection with ambiguous surfaces. Later the ideas where used by Ronda et al [10] for calibrating cameras.

On the other hand Opthalmologists have been interested in Horopters for much longer and still publish papers in this area, for example [13].

A slightly different problem with the same solution concerns a single camera subjected to a rigid-body motion. Clearly, the points in space which do not appear to have moved in the image, lie on the Horopter.

In this work the Horopter is studied using some ideas from line geometry. In particular, another old construction (though not quite as old): the complex of lines from successive points. If we join all points in space to their images under a given rigid-body motion, the set of lines we get lie in a quadratic line complex.

The connection between these two constructions is explored and modern proofs are given for many of the properties of these varieties. In particular the cases in which the Horopter can degenerate are found.

Finally, the infinitesimal case is considered, this has applications to optical flow.

J.M. Selig works for the Faculty of Business, Computing and Information Management, London South Bank University, London SE1 OAA, U.K. seligjmelsbu.ac.uk
In the next section a very brief introduction is given to line geometry. This serves to fix notation, a fuller account of the subject can be found in several places, most recently perhaps [7] or [12].

\section{LINE GEOMETRY}

Classical line geometry is the study of all lines in 3dimensional space. The set of all possible lines in space forms a four dimensional variety known as the Klein quadric.

To see this we introduce the Plücker coordinates of a line. Let $\mathbf{p}_{1}=\left(x_{1}, y_{1}, z_{1}\right)^{T}$ and $\mathbf{p}_{2}=\left(x_{2}, y_{2}, z_{2}\right)^{T}$ be the position vectors of a pair of points. The line joining them has direction $\mathbf{p}_{1}-\mathbf{p}_{2}$ and moment $\mathbf{p}_{1} \times \mathbf{p}_{2}$. The six quantities here are the Plücker coordinates of the line, they can be written as a 6-vector,

$$
\left(\begin{array}{c}
p_{01} \\
p_{02} \\
p_{03} \\
p_{23} \\
p_{31} \\
p_{12}
\end{array}\right)=\left(\begin{array}{c}
x_{2}-x_{1} \\
y_{2}-y_{1} \\
z_{2}-z_{1} \\
y_{1} z_{2}-z_{1} y_{2} \\
z_{1} x_{2}-x_{1} z_{2} \\
x_{1} y_{2}-y_{1} z_{2}
\end{array}\right)
$$

This will be written in the partitioned form,

$$
\left(\begin{array}{l}
\mathbf{p}_{1}-\mathbf{p}_{2} \\
\mathbf{p}_{1} \times \mathbf{p}_{2}
\end{array}\right)=\left(\begin{array}{c}
\boldsymbol{\omega} \\
\mathbf{v}
\end{array}\right) .
$$

The advantage of this parameterisation of lines is that if a different pair of (distinct) points are chosen on the line the Plücker coordinates only change by an overall multiplicative factor. Hence, these 6-vectors should be thought of as homogeneous coordinates in a 5-dimensional projective space $\mathbb{P}^{5}$. Not every point in $\mathbb{P}^{5}$ represents a line, only those which satisfy the homogeneous quadratic relation $\boldsymbol{\omega} \cdot \mathbf{v}=0$. The points satisfying this equation form a 4-dimensional subspace in $\mathbb{P}^{5}$ usually called the Klein quadric. The 2-plane of points in the Klein quadric satisfying $\boldsymbol{\omega}=\mathbf{0}$ do not represent lines in space, they are called infinite lines.

Notice that the equation for the Klein quadric could have been written,

$$
\left(\boldsymbol{\omega}^{T}, \mathbf{v}^{T}\right)\left(\begin{array}{cc}
0 & I_{3} \\
I_{3} & 0
\end{array}\right)\left(\begin{array}{l}
\boldsymbol{\omega} \\
\mathbf{v}
\end{array}\right)=0
$$

where $I_{3}$ is the $3 \times 3$ identity matrix. In the following the matrix $\left(\begin{array}{cc}0 & I_{3} \\ I_{3} & 0\end{array}\right)$ will be denoted $Q_{0}$. For two lines $\mathbf{l}_{1}$ and $\mathrm{l}_{2}$ we can form the symmetric product,

$$
\mathbf{l}_{1}^{T} Q_{0} \mathbf{l}_{2}=\boldsymbol{\omega}_{1} \cdot \mathbf{v}_{2}+\mathbf{v}_{1} \cdot \boldsymbol{\omega}_{2} .
$$

This product is invariant under the action of the group of rigid-body motions. That is its value will be the same if 
both lines are subjected to the same rigid-body motion or if the product is evaluated in different orthogonal coordinate systems. Geometrically its value is given by the product of the perpendicular distance between the lines times the sine of the angle between them. The product is zero if and only if the lines meet or are parallel. Two lines which satisfy $\mathbf{l}_{1}^{T} Q_{0} \mathbf{l}_{2}=0$ are said to be reciprocal.

There is another invariant product on the lines,

$$
\mathbf{l}_{1}^{T} Q_{\infty} \mathbf{l}_{2}=\left(\boldsymbol{\omega}_{1}^{T}, \mathbf{v}_{1}^{T}\right)\left(\begin{array}{cc}
I_{3} & 0 \\
0 & 0
\end{array}\right)\left(\begin{array}{l}
\boldsymbol{\omega}_{2} \\
\mathbf{v}_{2}
\end{array}\right)=\boldsymbol{\omega}_{1} \cdot \boldsymbol{\omega}_{2}
$$

This matrix $\left(\begin{array}{cc}I_{3} & 0 \\ 0 & 0\end{array}\right)$, will be denoted $Q_{\infty}$. This is known as the Killing form and represents the cosine of the angle between the lines.

The intersection of the Klein quadric with a hyperplane in $\mathbb{P}^{5}$ forms a 3-dimensional set of lines called a linear line complex. The lines in the linear line complex l satisfy a linear equations of the form,

$$
\mathbf{z}^{T} Q_{0} \mathbf{l}=0
$$

where $\mathbf{z}$ is a fixed 6-vector, not necessarily a line. Such vectors will be called screws here.

A quadratic line complex is the intersection in $\mathbb{P}^{5}$, of the Klein quadric with a degree 2 hypersurface also called a quadric. The lines 1 , lying in in a quadratic line complex satisfy a quadratic equation,

$$
\mathbf{l}^{T} K \mathbf{l}=0
$$

where $K$ is a symmetric $6 \times 6$ matrix. See [5] for much more on the subject of line complexes.

\section{A QuAdratic Line COMPLEX}

Suppose that $\mathbf{p}_{1}$ is a point in space, now we subject this to a rigid-body motion. The original point will be transformed to a new position,

$$
\mathbf{p}_{2}=R \mathbf{p}_{1}+\mathbf{t}
$$

In general, these two points will be different and so it will be possible to produce a line through both points, see Fig. 1. As $\mathbf{p}_{1}$ ranges over all points in space, keeping the rigid transformation fixed, we obtain a family of lines. It is well known that this family of lines comprise a quadratic line complex, see [2, chap.3 §3]. Here a different derivation is given, one which leads directly to a coordinate free description of the $6 \times 6$ symmetric matrix of the complex.

Begin by assuming that the finite screw motion is about a line $\mathbf{l}$ through a rotation angle $\theta$ and with a pitch $h$. That is a rotation by $\theta$ about 1 followed by a translation of $\theta h / 2 \pi$ along $\mathbf{l}$. Without loss of generality we can assume that, $\mathbf{p}_{1}=$ $(d, 00)^{T}$ and

$$
\mathbf{l}=\left(\begin{array}{c}
\lambda \mathbf{k} \\
\mathbf{0}
\end{array}\right)
$$

where $d$ is the perpendicular distance of $\mathbf{p}_{1}$ from the screw axis $\mathbf{l}, \mathbf{k}$ is the unit vector in the $z$-direction and $\lambda$ is a non-zero constant with $\lambda^{2}=\mathbf{l}^{T} Q_{\infty}$ l. Generality is not lost because it is always possible to bring the axis and point into this configuration using a suitable rigid transformation. After the rigid motion we have,

$$
\mathbf{p}_{2}=\left(\begin{array}{c}
d \cos \theta \\
d \sin \theta \\
\theta h / 2 \pi
\end{array}\right)
$$

Hence the line joining $\mathbf{p}_{1}$ and $\mathbf{p}_{2}$ will have Plücker coordinates proportional to,

$$
\mathbf{s}=\left(\begin{array}{c}
\mathbf{p}_{1}-\mathbf{p}_{2} \\
\mathbf{p}_{1} \times \mathbf{p}_{2}
\end{array}\right)=\left(\begin{array}{c}
d(1-\cos \theta) \\
-d \sin \theta \\
-\theta h / 2 \pi \\
0 \\
-d \theta h / 2 \pi \\
d^{2} \sin \theta
\end{array}\right)
$$

Although the above has been computed using a particular values for the axis $\mathbf{l}$ and point $\mathbf{p}_{1}$, if we compute invariant quantities linking $\mathbf{l}$ and $\mathbf{p}_{1}$ the results will not depend on this particular position. We could subject the line and point to a rigid transformation and the results would be the same. The possible invariants involving $\mathbf{l}$ and $\mathrm{s}$ are,

$$
\mathbf{l}^{T} Q_{\infty} \mathbf{s}=-\theta h \lambda / 2 \pi, \quad \mathbf{l}^{T} Q_{0} \mathbf{s}=d^{2} \lambda \sin \theta
$$

and

$$
\mathbf{s}^{T} Q_{\infty} \mathbf{s}=2 d^{2}(1-\cos \theta)+\theta^{2} h^{2} / 4 \pi^{2} .
$$

We can use these results to eliminate $d$, the only quantity specific to $\mathbf{p}_{1}$. We must be a little careful here since $\mathbf{s}$ is only proportional to the line joining $\mathbf{p}_{1}$ to $\mathbf{p}_{2}$, if we ensure that the expression we produce is homogeneous in the components of $\mathbf{s}$ then this will not matter. It is easy to see that such an expression is given by,

$$
\frac{\left(\mathbf{l}^{T} Q_{\infty} \mathbf{s}\right)^{2}-\lambda^{2} \mathbf{s}^{T} Q_{\infty} \mathbf{s}}{\left(\mathbf{l}^{T} Q_{0} \mathbf{s}\right)\left(\mathbf{l}^{T} Q_{\infty} \mathbf{s}\right)}=\frac{4 \pi(1-\cos \theta)}{\theta h \sin \theta}=\frac{2 \pi \tan (\theta / 2)}{h(\theta / 2)} .
$$

The quantity $h(\theta / 2) / 2 \pi \tan (\theta / 2)$ will be abbreviated to $q$ following Parkin and Hunt's quasi-pitch or 'quatch', [6]. So the above equation can be written as,

$$
\left(\mathbf{l}^{T} Q_{0} \mathbf{s}\right)\left(\mathbf{l}^{T} Q_{\infty} \mathbf{s}\right)+q\left(\lambda^{2}\left(\mathbf{s}^{T} Q_{\infty} \mathbf{s}\right)-\left(\mathbf{l}^{T} Q_{\infty} \mathbf{s}\right)^{2}\right)=0 .
$$

The symmetric matrix $K$, of the quadratic complex can be seen to be,

$$
K=q \lambda^{2} Q_{\infty}-q Q_{\infty} \mathbf{l l}^{T} Q_{\infty}+\frac{1}{2} Q_{0} \mathbf{l l}^{T} Q_{\infty}+\frac{1}{2} Q_{\infty} \mathbf{l l}^{T} Q_{0} .
$$

Now, we may assume that the 1 is a general line, and we can write it as,

$$
\mathbf{l}=\left(\begin{array}{l}
\omega \\
\mathbf{v}
\end{array}\right)
$$

with $\boldsymbol{\omega} \cdot \mathbf{v}=0$, then the symmetric matrix $K$ can be written, $K=q\left(\begin{array}{cc}(\boldsymbol{\omega} \cdot \boldsymbol{\omega}) I_{3}-\boldsymbol{\omega} \boldsymbol{\omega}^{T} & 0 \\ 0 & 0\end{array}\right)+\frac{1}{2}\left(\begin{array}{cc}\boldsymbol{\omega} \mathbf{v}^{T}+\mathbf{v} \boldsymbol{\omega}^{T} & \boldsymbol{\omega} \boldsymbol{\omega}^{T} \\ \boldsymbol{\omega} \boldsymbol{\omega}^{T} & 0\end{array}\right)$,

where $\lambda^{2}$ has been replaced with $\mathbf{l}^{T} Q_{\infty} \mathbf{l}=\boldsymbol{\omega} \cdot \boldsymbol{\omega}$.

Now it is well known that there is a correspondence between 3 -vectors and $3 \times 3$ anti-symmetric matrices. For 


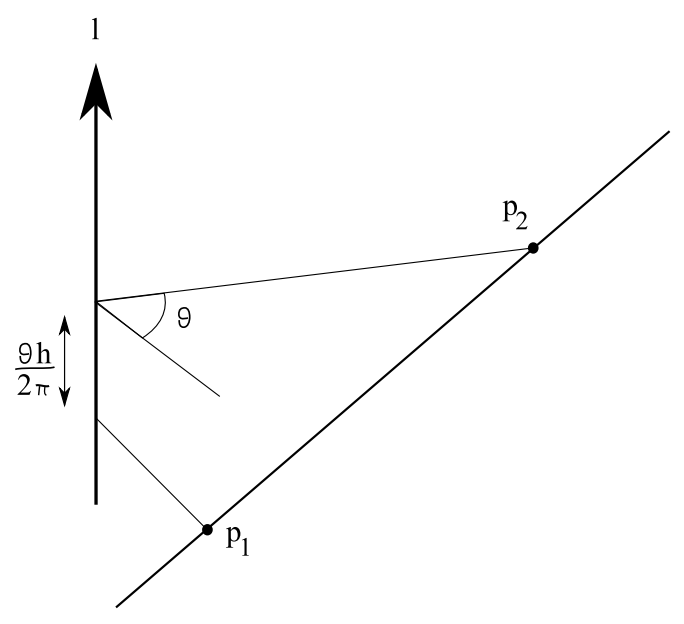

Fig. 1. A Line From the Complex of Lines from Successive Points.

example the anti-symmetric matrix corresponding to $\boldsymbol{\omega}=$ $\left(\omega_{x}, \omega_{y}, \omega_{z}\right)^{T}$ is,

$$
\Omega=\left(\begin{array}{ccc}
0 & -\omega_{z} & \omega_{y} \\
\omega_{z} & 0 & -\omega_{x} \\
-\omega_{y} & \omega_{x} & 0
\end{array}\right)
$$

From this we have that, $\Omega \mathbf{p}=\boldsymbol{\omega} \times \mathbf{p}$ for and vector $\mathbf{p}$. Given two anti-symmetric matrices $X$ and $Y$ corresponding to vectors $\mathbf{x}$ and $\mathbf{y}$ respectively, it is easy to verify the following relation; $X Y=\mathbf{y} \mathbf{x}^{T}-(\mathbf{x} \cdot \mathbf{y}) I_{3}$. This can be used to write the symmetric matrix $K$ as,

$$
K=q\left(\begin{array}{cc}
-\Omega^{2} & 0 \\
0 & 0
\end{array}\right)+\frac{1}{2}\left(\begin{array}{cc}
\Omega V+V \Omega & \Omega^{2}+I_{3} \\
\Omega^{2}+I_{3} & 0
\end{array}\right) .
$$

Since lines lie on the Klein quadric any multiple of $Q_{0}$ can be subtracted from $K$ without effect, so the symmetric matrix of this quadratic line complex can be written,

$$
K=q\left(\begin{array}{cc}
-\Omega^{2} & 0 \\
0 & 0
\end{array}\right)+\frac{1}{2}\left(\begin{array}{cc}
\Omega V+V \Omega & \Omega^{2} \\
\Omega^{2} & 0
\end{array}\right) .
$$

Notice that transformations with the same axis and quatch produce the same quadratic complex of lines.

If we let $\mathbf{p}_{0}$ be an arbitrary point in space then the lines in the complex through $\mathbf{p}_{0}$ form a quadric cone with vertex $\mathbf{p}_{0}$. To see this observe that the lines passing through the point $\mathbf{p}_{0}$ have the general form,

$$
\left(\begin{array}{c}
\mathbf{u} \\
\mathbf{p}_{0} \times \mathbf{u}
\end{array}\right)=\left(\begin{array}{c}
I_{3} \\
P_{0}
\end{array}\right) \mathbf{u}
$$

where $\mathbf{u}$ is an arbitrary non-zero vector, representing the direction of the line. Substituting this into the quadratic complex (10), gives a quadratic equation for $\mathbf{u}$,

$$
\mathbf{u}^{T}\left(-2 q \Omega^{2}+\Omega V+V \Omega+\Omega P_{0}-P_{0} \Omega\right) \mathbf{u}=0,
$$

so the set of possible directions $\mathbf{u}$, for the lines through $\mathbf{p}_{0}$ lie on a conic.

\section{THE HOROPTER}

As mentioned in the introduction above there are two related problems here. In one problem there is a single camera and the objects undergo a rigid motion $g$. So consider a 'pinhole' camera with optical centre located at $\mathbf{p}_{0}$. The set of points $\mathbf{p}$ such that $\mathbf{p}$ and $g(\mathbf{p})=R \mathbf{p}+\mathbf{t}$ produce the same image point form the Horopter. Clearly this means that $\mathbf{p}_{0}, \mathbf{p}$ and $g(\mathbf{p})$ will be co-linear.

The original problem is concerned with stereo vision. Here we have two cameras with optical centres $\mathbf{p}_{0}$ and $g^{-1}\left(\mathbf{p}_{0}\right)=R^{T} \mathbf{p}_{0}-R^{T} \mathbf{t}$. If an image point is $\mathbf{p}$ then it lies on the Horopter if $\mathbf{p}-\mathbf{p}_{0}$ and $g\left(\mathbf{p}-g^{-1}\left(\mathbf{p}_{0}\right)\right)=$ $g(\mathbf{p})-\mathbf{p}_{0}$ lie in the same direction. Clearly this is the same problem as the one above however notice that if we actively transform the objects by a transformation $g$, the corresponding transformation of the cameras will be by the inverse $g^{-1}$. Sometimes it is easier to think of this problem in terms of stereo vision and sometimes in terms of a motion of space, both views will be referred to freely in the following.

Notice that the model of the camera being used here is very simple. The points in the image are represented by lines through the optical centre of the camera. To produce actual coordinates for the image plane it would be enough to distinguish one of these lines as the optical axis of the camera. However, it is not necessary to do this here.

The set of all lines joining $\mathbf{p}$ and $g(\mathbf{p})$ form the quadratic line complex described above. The lines which also pass through the point $\mathbf{p}_{0}$ form a quadric cone with vertex $\mathbf{p}_{0}$, as we have also seen. Now we can also consider the points $\mathbf{p}$ and $R^{T} \mathbf{p}-R^{T} \mathbf{t}=g^{-1}(\mathbf{p})$. The lines through these points lie on a quadric cone with vertex $g^{-1}\left(\mathbf{p}_{0}\right)$ associated with the quadratic line complex for the rigid transformation $g^{-1}$. This is simply the rigid transformation of the original cone by the inverse of the transformation under consideration. It also contains the Horopter, but it is also easy to see that the line joining the vertices of the two cones $\mathbf{p}_{0}$ and $g^{-1}\left(\mathbf{p}_{0}\right)$, also lies in both cones. This line joins the two optical centres and is usually called the epipolar line.

Two quadric cones intersect in a degree four curve in 
general. Here we see that the intersection contains a line, hence the quartic is not irreducible, the residual curve must be a cubic. When this cubic curve is irreducible it will necessarily be a twisted cubic, that is a rational normal cubic curve, [3, Chap. I].

A simple consequence of this construction is that the image of the Horopter, that is its projection to the image plane of either camera, will be a conic curve given by (11).

Another way to view the Horopter is as the intersection of three quadrics. The condition that the points $\mathbf{p}_{0}, \mathbf{p}$ and $g(\mathbf{p})$ should be co-linear can be stated as,

$$
\left(\mathbf{p}-\mathbf{p}_{0}\right) \times\left(R \mathbf{p}+\mathbf{t}-\mathbf{p}_{0}\right)=\mathbf{0} .
$$

This vector equation contains three quadratic equations in the components of $\mathbf{p}$. From this equation it is easy to see that the points $\mathbf{p}=\mathbf{p}_{0}$ and $\mathbf{p}=R^{T} \mathbf{p}_{0}-R^{T} \mathbf{t}$ lie on all three quadrics and hence on the Horopter. That is, the Horopter passes through the optical centres of the cameras and so the epipolar line is a chord.

A defining property of the twisted cubic curve is that it can be parameterised by cubic polynomials, see for example [11, Chap. XII]. A parameterisation for the Horopter can be found as follows. To begin with the position vectors of points will be written as,

$$
\tilde{\mathbf{p}}=\left(\begin{array}{c}
\mathbf{p} \\
1
\end{array}\right), \quad \tilde{\mathbf{p}}_{0}=\left(\begin{array}{c}
\mathbf{p}_{0} \\
1
\end{array}\right)
$$

and so forth. This is so that the homogeneous representation of the group can be used, that is $g(\mathbf{p})$ will be represented by $G \tilde{\mathbf{p}}$ where,

$$
G=\left(\begin{array}{cc}
R & \mathbf{t} \\
0 & 1
\end{array}\right)
$$

Equation (12) can be written as,

$$
\lambda\left(\tilde{\mathbf{p}}-\tilde{\mathbf{p}}_{0}\right)=\mu\left(G \tilde{\mathbf{p}}-\tilde{\mathbf{p}}_{0}\right),
$$

introducing parameters $\lambda$ and $\mu$. Rearranging this equations we obtain,

$$
\left(\mu G-\lambda I_{4}\right) \tilde{\mathbf{p}}=(\mu-\lambda) \tilde{\mathbf{p}}_{0} .
$$

Now, think of the components of the vectors as homogeneous coordinates, that is think of space as a subset of projective space $\mathbb{P}^{3}$ with coordinates $\tilde{\mathbf{p}}^{T}=(x: y: z: w)$. In these homogeneous coordinates multiplication by a common fact has no effect, so we can ignore factors like $(\mu-\lambda)$ and $\operatorname{det}\left(\mu G-\lambda I_{4}\right)$. The cubic parameterisation can then be written,

$$
\tilde{\mathbf{p}}=\operatorname{Adj}\left(\mu G-\lambda I_{4}\right) \tilde{\mathbf{p}}_{0},
$$

where Adj denotes the adjugate of the matrix, that is the transposed matrix of cofactors. Since the cofactors of a $4 \times 4$ matrix are $3 \times 3$ determinants the elements of the adjugate, and hence the coordinates of $\tilde{\mathbf{p}}$, are indeed cubic functions of the parameters $\lambda$ and $\mu$. Notice that the optical centres of the two cameras are given by the parameter values $\mu=0, \lambda \neq 0$ and $\mu \neq 0, \lambda=0$.

\section{Cubical Ellipses}

Up to projective transformations there is just one twisted cubic curve. However, if we are only interested in rigid-body transformation the twisted cubic curves can be classified according to how they meet the plane at infinity and the circle at infinity, [14], [15].

It can be shown that the Horopter meets the plane at infinity at three points, one real and a pair of complex conjugate points. To see this we can choose coordinates in space so that $\mathbf{p}_{0}$ is located at the origin and the $x$-axis is aligned with the axis of the rotation matrix $R$. Writing $\mathbf{p}^{T}$ as $(x, y, z)$ and re-introducing the variable $w$ to make the equations homogeneous, (12) becomes,

$$
\begin{aligned}
\left(\begin{array}{l}
x \\
y \\
z
\end{array}\right) \times\left(\left(\begin{array}{ccc}
1 & 0 & 0 \\
0 & \cos \theta & -\sin \theta \\
0 & \sin \theta & \cos \theta
\end{array}\right)\left(\begin{array}{l}
x \\
y \\
z
\end{array}\right)+w\left(\begin{array}{l}
t_{x} \\
t_{y} \\
t_{z}
\end{array}\right)\right) \\
=\left(\begin{array}{l}
0 \\
0 \\
0
\end{array}\right),(16)
\end{aligned}
$$

where $\theta$ is the rotation angle of the transformation and $t_{x}, t_{y}$ and $t_{z}$ are the components of the translation vector. Setting $w=0$ will give the intersections of the Horopter with the plane at infinity. The equation above becomes,

$$
\mathbf{p} \times R \mathbf{p}=\mathbf{0} .
$$

This obviously has three solutions corresponding to the three eigenvectors of $R$. The real solution is $\mathbf{p}^{T}=(1,0,0)$ corresponding to the axis of the rotation, the complex solutions are $\mathbf{p}^{T}=(0,1, i)$ and $\mathbf{p}^{T}=(0,1,-i)$ where $i$ is the imaginary unit. Further the complex solutions clearly lie on $x^{2}+y^{2}+z^{2}=0$, the imaginary circle at infinity. These results do not depend on the choice of coordinate frame so will be true in general. Hence the Horopter is a special cubical ellipse [15].

It is well known that such a curve must lie on a circular cylinder. Moreover, this circular cylinder must lie in the net of quadrics (12). A particular quadric in this net can be selected by taking the scalar product of (12) with a constant vector. To make things a little easier, let $\mathbf{p}_{0}$ be the origin of coordinates now, and use the constant unit vector $\boldsymbol{\omega}$ corresponding to the rotation axis of $R$,

$$
\boldsymbol{\omega} \cdot \mathbf{p} \times(R \mathbf{p}+\mathbf{t})=-\mathbf{p}^{T} \Omega(R \mathbf{p}+\mathbf{t})=0,
$$

where $\Omega$ is the $3 \times 3$ anti-symmetric matrix corresponding to $\boldsymbol{\omega}$, as above. The rotation matrix $R$ can be written,

$$
R=I_{3}+\sin \theta \Omega+(1-\cos \theta) \Omega^{2},
$$

using the well known Rodrigues formula. This leads to,

$$
\sin \theta\left(\mathbf{p}^{T} \Omega^{2} \mathbf{p}\right)+\mathbf{p}^{T} \Omega \mathbf{t}=0
$$

since $\Omega$ and $\Omega^{3}=-\Omega$ are anti-symmetric. To see that this quadric is indeed a circular cylinder choose coordinate such that $\boldsymbol{\omega}$ is aligned along $x$-axis. The quadric now becomes,

$$
-\sin \theta\left(y^{2}+z^{2}\right)+z t_{y}-y t_{z}=0 .
$$


Clearly this is a circular cylinder with, axis given by the line $y=-t_{z} / 2 \sin \theta, z=t_{y} / 2 \sin \theta$ and radius $\left(t_{y}^{2}+t_{z}^{2}\right) / 4 \sin ^{2} \theta$.

The screw axis of the motion lies on this cylinder, suppose $\mathbf{a}$ is a point on the screw axis. The effect of the transformation on such a point will be, $g(\mathbf{a})=R \mathbf{a}+\mathbf{t}=$ $\mathbf{a}+(\theta h) /(2 \pi) \boldsymbol{\omega}$, that is a translation along the screw axis by $(\theta h) /(2 \pi)$ where $h$ is the pitch of the screw. Substituting this into the equation for the cylinder (18) gives,

$$
\mathbf{a}^{T} \Omega(R \mathbf{a}+\mathbf{t})=\mathbf{a} \cdot\left(\boldsymbol{\omega} \times\left(\mathbf{a}+\frac{\theta h}{2 \pi} \boldsymbol{\omega}\right)\right)=0 .
$$

Since this vanishes identically, all points on the screw axis lie on the cylinder. The screw axis is in fact tangent to the Horopter at infinity and hence is the asymptote for the curve, see Fig. 2 .

\section{SINGULARITIES}

As mentioned above, the image of the Horopter in either of the cameras is a conic. However, this conic can be degenerate. This can happen in two possible ways. Either the motion $g$ is such that the Horopter itself degenerates. Alternatively, the optical centre of the camera may be located at a point where the conic degenerates. Recall that the conic is determined by the $3 \times 3$ symmetric matrix,

$$
M=-2 q \Omega^{2}+\Omega V+V \Omega+\Omega P_{0}-P_{0} \Omega,
$$

see (11) above. The conic will be degenerate if $\operatorname{det}(M)=$ 0 . This determinant will be a function of the rigid-body motion $g$ and the coordinates of the optical centre $\mathbf{p}_{0}$. If the Horopter itself is degenerate then $\operatorname{det}(M)$ will vanish for all possible values of $\mathbf{p}_{0}$. Again here we can choose a coordinate system to simplify our computations, suppose we choose a coordinate frame whose origin is located on the screw axis of the motion, this will make the anti-symmetric matrix $V$ vanish. Further we can choose the $x$-axis of our coordinate system to be aligned with the screw axis of the motion, so that,

$$
\Omega=\left(\begin{array}{ccc}
0 & 0 & 0 \\
0 & 0 & -1 \\
0 & 1 & 0
\end{array}\right), \quad \text { and } \quad P_{0}=\left(\begin{array}{ccc}
0 & -z & y \\
z & 0 & -x \\
-y & x & 0
\end{array}\right)
$$

is the position of the optical centre.

In this coordinate frame the determinant becomes,

$$
\operatorname{det}(M)=2 q\left(y^{2}+z^{2}\right) .
$$

For a general quadratic line complex this determinant would be a quartic in the coordinates of $\mathbf{p}_{0}$, representing a degree four surface in space usually know as the Kummer quartic surface, [5, Chap. VI]. In special cases, this surface degenerates to four planes, such a quadratic line complex is called a tetrahedral line complex. In the case under consideration we see that, for non-zero $q$, the complex is tetrahedral, the four singular planes being given by $y=i z$, $y=-i z$ and the plane at infinity counted twice. The finite, complex conjugate planes intersect in the real line $y=z=0$ which is just the screw axis of the motion. That is, for any rigid-motion the image of the Horopter will be degenerate if the optical centre of the cameras lie on the screw axis of the motion.

If the quatch $q$, of the motion is zero then the Horopter itself will be degenerate. Since $q=h(\theta / 2) / 2 \pi \tan (\theta / 2)$, this can happen if the pitch $h$, of the motion is zero or if the angle $\theta= \pm \pi$.

When $h=0$ the motion is a pure rotation. Now the rotation axis is part of the Horopter, since if a lies on the rotation axis $R \mathbf{a}+\mathbf{t}=\mathbf{a}$ and so $R \mathbf{a}+\mathbf{t}-\mathbf{p}_{0}=\mathbf{a}-\mathbf{p}_{0}$ satisfying (12). The residual curve will be a conic, in fact a circle since it meets the circle at infinity in a pair of complex conjugate points. This circle has a long history in the study of binocular vision dating back to the early 1800 s, it is known as the Vieth-Muller circle.

If the rotation angle is $\pm \pi$ then the cameras are facing in opposite directions and we don't expect to see anything. Mathematically, the Horopter in this case contains a line at infinity, the residual curve is a hyperbola in the plane containing the two optical centres and the screw axis of the motion.

The final case to consider is when the motion is a pure translation, this has not been considered so far since in this case the pitch is usually said to infinite. However, it is easy to see in this case that the Horopter is no longer a curve but consists of all the points on the plane at infinity.

\section{OPTICAL FLOW}

A closely related problem concerns optical flow. The optical flow is an approximation to the projection of an object point's velocity onto the image plane of a single camera. So now we are dealing with infinitesimal motions rather that finite motions as above. It is reasonable to ask which points in space have velocities that cannot be detected in the image. The velocities of these points will be directed along the line joining the point to the optical centre of the camera. Again the these points form a twisted cubic. The velocity of point $\mathbf{p}$ will be $\boldsymbol{\omega} \times \mathbf{p}+\mathbf{w}$ where now $\boldsymbol{\omega}$ is the angular velocity and $\mathbf{w}$ is the linear velocity of the origin. Using homogeneous coordinates we get an equation very similar to (13),

$$
\lambda\left(\tilde{\mathbf{p}}-\tilde{\mathbf{p}}_{0}\right)=\mu S \tilde{\mathbf{p}}
$$

where,

$$
S=\left(\begin{array}{cc}
\Omega & \mathbf{w} \\
0 & 0
\end{array}\right)
$$

is the instantaneous velocity screw of the motion. So by the same argument as above,

$$
\tilde{\mathbf{p}}=\operatorname{Adj}\left(\mu S-\lambda I_{4}\right) \tilde{\mathbf{p}}_{0},
$$

gives a rational cubic parameterisation of the curve. This twisted cubic curve shares many properties of the Horopter, for example since $\Omega$ has the same eigenvectors as the corresponding rotation matrix $R=e^{\Omega}$, this twisted cubic is also a cubical ellipse lying on a circular cylinder.

In this situation we can also find a quadratic complex of lines. Take a point $\mathbf{p}$, now construct the line through this point aligned with velocity of the point $\boldsymbol{\omega} \times \mathbf{p}+\mathbf{w}$. The set 


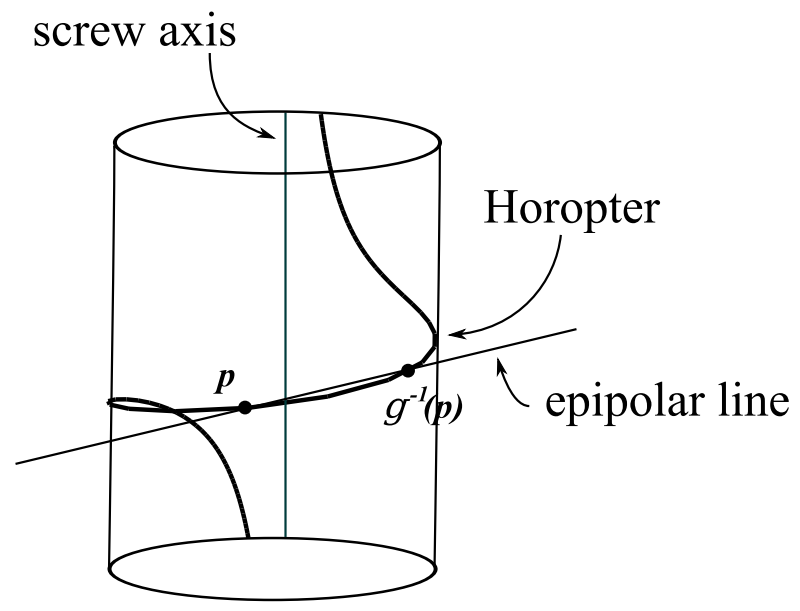

Fig. 2. The Horopter lies on a Circular Cylinder with the Screw axis as Asymptote.

of all such lines for each point in space form a quadratic line complex. This can be seen by following the argument given in section III above. The symmetric matrix of the complex is therefore,

$$
K=h\left(\begin{array}{cc}
-\Omega^{2} & 0 \\
0 & 0
\end{array}\right)+\frac{1}{2}\left(\begin{array}{cc}
\Omega V+V \Omega & \Omega^{2} \\
\Omega^{2} & 0
\end{array}\right),
$$

where $\left(\begin{array}{l}\boldsymbol{\omega} \\ \mathbf{v}\end{array}\right)$ is the axis of the motion's infinitesimal screw. That is, where $\mathbf{w}=\mathbf{v}+h \boldsymbol{\omega}$ and $V$ is the anti-symmetric matrix corresponding to $\mathbf{v}$ in the usual way.

Notice that this is same matrix as for a finite motion but with the quatch $q$ replaced by the pitch $h$. Of course the factor of $1 / 2$ is irrelevant since the equation for the complex is homogeneous. This shows us that the image of the twisted cubic is again a conic in general.

\section{CONCLUSION}

Although much of this work is well known it has been presented using modern Lie group theory and a little algebraic geometry. This makes it more accessible to a modern audience and links it with other modern work in this area.

However, there are some new observations, for example in section VI the circumstances in which the Horopter and its image degenerate are studied. The novelty here is that it is clear that these are the only ways in which these curves can degenerate.

In section III it was observed that motions with the same axis and quatch produce the same quadratic complex. This means that such motions cannot be distinguished by observing the images of their Horopters whatever camera position is chosen.

The quadratic line complexes introduced in section III appears in other guises in Kinematics. It is the set of lines which are reciprocal to their images under a rigidbody motion and also the intersection of planes with their images under a rigid-body motion, [2, Chap.3]. The complex described in section VII can be thought of as the limiting case of the first complex as the rigid-body motion tends to an infinitesimal screw motion. It also appears as the complex of Helitangent lines introduced by Jack Phillips, [8, Chap. $11]$.

Twisted cubic curves also turn up in other places in Kinematics. In a continuous rigid motion points which are instantaneously at inflection points of their trajectories form a twisted cubic curve, [1].

In future work it is intended that more of this fascinating geometry can be explored and it applications to robotics uncovered.

\section{REFERENCES}

[1] E.H. Bokelberg, K.H. Hunt and P.R. Ridley, "Spatial Motion I Points of Inflection and the Differential Geometry of Screws", Mech Mach. Theory, 27(1):1-15, 1992.

[2] O. Bottema and B. Roth. Theoretical Kinematics. Dover Publications, New York, 1990.

[3] R. Hartshorne, Algebraic Geometry, Springer Verlag, New York, 1995.

[4] H.L.F. von Helholtz, "Handbuch der Physiologischen Optik" vol. 3, Leopold Voss, Leipzig, 1867. English edition "Phisiological Optics" ed. J.P.C. Southall, Optical Soc. America, 1925.

[5] C.M. Jessop, A Treatise on the Line Complex, Cambridge University Press, Cambridge, 1903.

[6] K.H. Hunt and I.A. Parkin, "Finite displacements of points, planes, and lines via screw theory", Mech. Mach. Theory 30(2):177-192, 1995.

[7] H. Pottmann and J. Wallner, Computational Line Geometry, Springer Verlag, Berlin, 2001.

[8] J. Phillips, Freedom in Machinery vol. 2: Screw Theory Exemplified, Cambridge University Press, Cambridge, 1990.

[9] S.J. Maybank, "The Projective Geometry of Ambiguous Surfaces", Phil. Trans. R. Soc. Lond. 332:1-47, 1990.

[10] J. I. Ronda, A. Valdés and F. Jaureguizar, "Camera Autocalibration and Horopter Curves", Int. J. Computer Vision 57(3):219-232, 2004.

[11] J.G Semple and G.T Kneebone. Algebraic Projective Geometry. Oxford University Press, Oxford, 1952 (Reissued 1998).

[12] J.M. Selig. Geometric Fundamentals of Robotics. Springer Verlag, New York, 2005.

[13] H. Solomons, "Derivation of the space horopter", Br. J. Physiol. Opt. 30(2-4):56-80, 1975.

[14] D.M.Y. Sommerville, Analytical Geometry of Three dimensions, Cambridge University Press, Cambridge 1934.

[15] P.W. Wood, The Twisted Cubic, Cambridge Tracts in Mathematics and Mathematical Physics vol. 14, Cambridge University Press, Cambridge, 1913. 